\title{
General Framework for Animal Food Safety Traceability Using GS1 and RFID
}

\author{
Weizhu Cao, Limin Zheng, Hong Zhu*, and Ping Wu \\ College of Information and Electrical Engineering, China Agricultural University, \\ Beijing, P.R. China, 100083 \\ Tel.: +86-13693562232 \\ zhuhongxie@cau.edu.cn
}

\begin{abstract}
GS1 is global traceability standard, which is composed by the encoding system (EAN/UCC, EPC), the data carriers identified automatically (bar codes, RFID), electronic data interchange standards (EDI, XML). RFID is a non-contact, multi-objective automatic identification technique. Tracing of source food, standardization of RFID tags, sharing of dynamic data are problems to solve urgently for recent traceability systems. The paper designed general framework for animal food safety traceability using GS1 and RFID. This framework uses RFID tags encoding with EPCglobal tag data standards. Each information server has access tier, business tier and resource tier. These servers are heterogeneous and distributed, providing user access interfaces by SOAP or HTTP protocols. For sharing dynamic data, discovery service and object name service are used to locate dynamic distributed information servers.
\end{abstract}

Keywords: animal food safety traceability, GS1, RFID, general framework.

\section{Introduction}

Many countries had suffered disasters and economic loss because of the diseases occurred in recent years such as Bovine Spongiform Encephalopathy(BSE), Dioxin Toxicosis, Clenbnterol Hydrochloride, foot and mouth disease (FMD), and these diseases make the whole world focus on the issue of food safety(Zhao Jinyan et al., 2008). The European Union, the United States, Australia and other developed countries have introduced laws and regulations to make a mandatory requirement on animal food safety traceability (Zan Linsen et al., 2006). The strengthening of the supervision of animal food safety management is in favor of improving product value and market competitiveness.

GS1 represents a global standard (GS1 standard), a global system (the global identification system, GS1 System), and a global organization. It is responsible for establishing the standards of bar code and data exchange for the world's goods, and determine RFID standard by EPCglobal. GS1 is a global food standard for traceability, with components of coding systems (EAN/UCC, EPC), data carriers (bar code,

\footnotetext{
* Corresponding author.
} 
RFID) and data exchange (EDI, XML). "GS1 traceability standard" launched by GS1 retroactively supplied standard operating procedures for the design and implementation of food safety programs, which used successfully in beef, fish, vegetables and other foods, and such track and trace systems of food industry chains "from farm to fork" had established successfully (GS1, 2009).

Existing animal food traceability systems are starting from the slaughter, and cannot be achieved from "the source" (Kong Hongliang et al., 2004; C. Shanahan, 2009). Databases of food traceability systems can be centralized or hierarchical. Centralized databases can only save the limited information, without particular information of products, and are not flexible (C. Shanahan, 2009). While hierarchical database need to resolve the data sharing between the databases at all levels, and data replication will lead to redundancy (Zhao Jinyan et al., 2008). Compared with the bar code, RFID possesses of advantages such as non-contact, multi-objective automatic identification, which make it used in animal food safety monitoring more and more, but the standardization of RFID application needs to be improved (Ci Xinxin et al., 2007).

\section{General Framework of Traceability}

Animal food industry chains (supply chain, sales chain) are long, mainly including breeding, slaughtering, processing, logistics and sales. Applications of animal food safety traceability systems in monitoring animal food safety, includes not only the records and monitoring during the whole growth process of animals from birth to entering the slaughterhouse (feeding management, vaccine injection, disease treatment, feed used), but also the query for information of every stage, which is from consumers by the unique identifier of each animal, when animal products are send to the consumer market (supermarkets, etc.).

Realization of the whole process trace of animal food safety mainly depends on the effective documentation, efficient management and seamless transfer of the key information of each stage. RFID tag, the data carrier links the information flow to real object flow, and implements data exchange of all steps. Each step, products are not

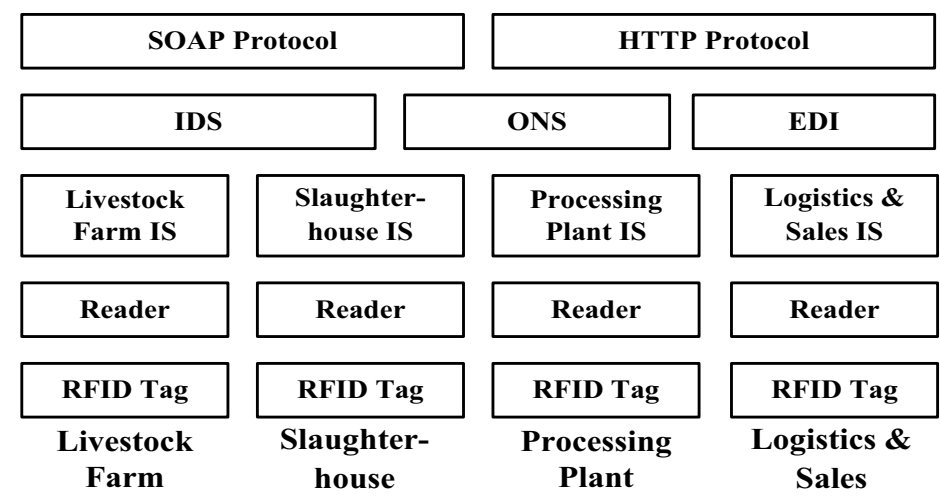

Fig. 1. General framework for animal food safety traceability 
the same form, and we need to identify these different forms of products. Synchronously, we use the unique identifier of each animal to associates each pattern of the products.

Taking GS1 standards and RFID identification technology and the characteristics of animal food industry chains into account, fully considering of the traceability systems at home and abroad, taking their public properties, we have designed the general framework of the animal food traceability, shown in Fig.1.

RFID is used to identify cattle individual, and we label different form product with different identification, such as using ear tags on farm to get the cattle information. RFID reader is used to achieve non-contact and multi-objective automatic identification, and improves collection efficiency of data. Because of geographical location, functions and other factors, breeding, slaughtering, processing, logistics and marketing, each basic stage builds their own information server (IS) to maintain the static and dynamic product information of the whole industry chain. These servers are heterogeneous and distributed, providing user access interfaces by SOAP or HTTP protocols. For tracing and tracking of each individual, each livestock is corresponding to a unique ID, and the information servers save the individual animal husbandry management, vaccine injection, disease treatment, feed used and other essential information. For sharing dynamic data, discovery service and object name service are used to locate dynamic distributed information servers.

\section{RFIDTAG}

Product identification is one fundamental aspect for food safety traceability. RFID is a non-contact, multi-objective automatic identification technique, widely used in traceability from farm to supermarket. RFID tags are low-capability devices, because cost and size requirements mean that power consumption, processing time, storage and logical gates are extremely limited.

EPCglobal Inc. has published the EPCglobal Tag Data Standard 1.4 for RFID tag contents (EPCglobal Inc. 2008). Its coding schemes include SGTIN, SSCC, SGLN, GRAI, GIAI, and so on.

In order to trace from farm to supermarket, a SGTIN (Serialized Global Trade Item Number) is used to identify individual logistical units (beef, feedstuff, medicine), a GLN (Global Location Number) is used to identify physical locations (farms, abattoirs, marts). In addition to a Header, the SGTIN-96 is composed of five fields: the Filter Value, Partition, Company Prefix, Item Reference, and Serial Number.

We propose that the EPC tag for goods identification contain the same identity number as the current ear tags, and a standard format for the content of the RFID tags should be compliant with the EPCglobal standard.

\section{Design and Implementation of Information Server}

\subsection{Design of Information Server}

Subject to capacity, RFID tags can only identify products, without the information of specific producing and the circulation. But consumers and suppliers want to query for 
various aspects of the industry chain and product information at all levels, which needs information servers to support it. Information Servers are used to record the process of producing and circulation, and provide users with such information service interfaces as capture and query. All sectors of the traceability system set up their own servers in accordance with the characteristics of the animal food chain. These servers are distributed, and even heterogeneous, different from centralized and hierarchical servers.

The information server saves the static and dynamic information of products throughout the industry chain. It provides static product-related data, when it belongs to the manufacturer's information system, such as manufacture date, validity period, raw materials and other related information. Participants provide dynamic databases for the management of the measured data and tracking data of mobile products, to provide such tracking content as delivery times, locations, transport ways.

Information server of each link is structured on TCP/IP network, distributed, even heterogeneous, with HTTP/SOAP to provide user with interfaces, and using XML to transfer message. Information servers of breeding, slaughtering, processing and other stages use C/S structure, which logistics and marketing stages B/S structure, supporting queries through the supermarket terminal, the Internet, and messaging platform.

\subsection{Implementation of Information Server}

Each information server has three-tier architecture (access tier, business tier and resource tier), in Fig.2. Access tier provides user access interface to information server, with the form of Web services, including the capture interface and query interface. Business tier is the core of information servers, implements specific capture processing, query processing, query callback interface, authentication, and pursue a variety of business rules and logics. Resource tier stores product management information to provide data to the business tier, using relational database. JAVA language is adopted for implementing information servers, MySQL 6.0 for databases, and ADO technology for data access.

In the business tier, authentication module completes authentication by the user name and password. Capture handling module bind Java Servlet with HTTP. When capture interface receives capture request of HTTP POST from the user, it binds the XML format for the capture message, and hands to the capture handling module. Capture handling module maps message captured with SQL statements, and stores in the database.

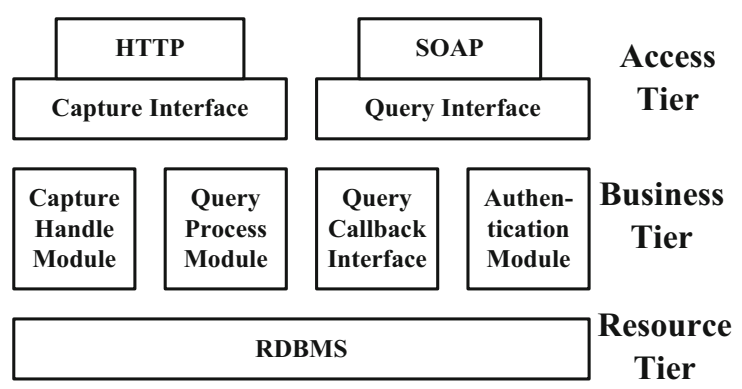

Fig. 2. Architecture of information server 
Query Processing uses Simple Object Access Protocol (SOAP) to package the message as Web service. SOAP makes different applications exchange information to with each other through the HTTP protocol, and by XML format. When query interface receives the SOAP request of XML format from the user, it binds XML to query parameters, and send to the query processing module. The query processing module generates SQL statements dynamically according to query parameters, query databases, and returns the results with the package of SOAP response of XML format. Users can reserve asynchronous query from callback interface. The asynchronous query is scheduled at a certain time or triggered by an event. And the returned query results call HTTP/HTTPS POST requests from the designated URL.

\section{Realization Mechanisms of the Dynamic Information Sharing}

\subsection{Dynamic Information Sharing}

Animal food chain involves several participants to work together, and each participant has its own Information Server. When recalling, or protecting the privacy, the producer might hope to track all information about the product. Because of geographical location, functions and other factors, each link of the animal food industry chain can configure for its own Information Server. These servers are geographically distributed, heterogeneous and cross-platform. In the industry chain, what specific supply links will the product pass is not known in advance, so the information server is dynamic. Data sharing between distributed and heterogeneous databases is the key problem to be solved in animal food safety.

Data transfer and data sharing are very important in animal food safety traceability. For example, in Ireland beef GS1 retrospective case (J. Brackenm et al., 2005), slaughterhouses and processing plants are in one company, share the same database system, while the sale is taken charge by another company, which has its own traceability system. When the cattle is carried to the slaughterhouse, the ID of cattle ear tag and some of the historical information are copied to the database of the slaughterhouse. Data synchronization and data redundancy are required to consider. Into the marketing chain, the bar code information of body division will be transmitted into the company's system of safety traceability. This case can only trace back to the farm name and location, without specific information of the cattle individual, such as the information of growth, feed, disease treatment and vaccine injection.

The distributed retrospective database query can be achieved while introducing the query engine (A. Cheung et al., 2007). Query engine queries the local database, and analyze the results. New query submitted to other databases on the network can be reconstructed, depend on whether the local search results are complete or not. Results returned from other databases are added to the local search results for post-processing, and returned to the user. In EPCglobal Network system, EPC Information Service provides a query interface to support simple local inquiries, but do not support crossorganization, distributed and complex queries of the database (EPCglobal Inc., 2009). Object name service (ONS) can support distributed database query, but the ONS is a static query engine, can only provide static information resources of product EPC coding. ONS uses the domain name system of Internet, has a single service model, 
updates slow, hasn't strong resource description, and security cannot be guaranteed (B. Fabian et al., 2005; S Beier, 2006).

\subsection{Implementation Mechanism}

In the animal food safety traceability general framework, Information Discovery Service and ONS are used to share data. The discovery service of distributed system responds to requests of inquiry for resources, locates them or find a number of locations of resources for cross-system. Information discovery service is quite a dynamic discovery engine to provide dynamic trace information.

Information services are provided by the database and a series of Web services interface. Database records include the following attributes:

1) Identification Code.

2) URL of the information server submitting records.

3) Certificate: the certificate of the company which owns the information server and has submitted the record.

4) Security Marker: specify whether the records can be read for all, or only for partners on the industrial chain

5) Timestamp: recording time.

Web service interface in the information discovery service is the interface for users to access. When the interface is connected to the Internet, it can be called by any computer on the Internet, but only those authorized computers can read and write the specific data elements. These Web service interfaces allow authorized companies to register a record when they produce or receive a new product. In addition, these authorized companies use identification code to enquire the URL of the Information Server saving the product information.

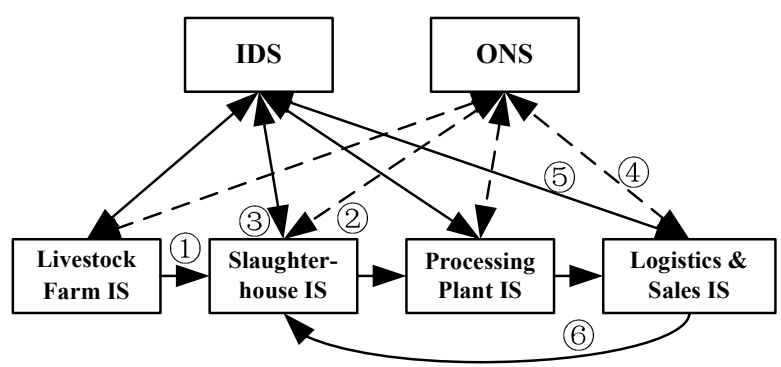

Fig. 3. Work principle of IDS and ONS

Before enterprises in animal food industry chain use discovery services, they need to apply for authorization to the authority to obtain a certificate. The information server configured register from the ONS static, the working principle in Fig.3. Registration process of dynamic information server to the IDS:

1) Animal food mobile in the industry chain, RFID readers in slaughterhouses identifying need for registration to the IDS; 
2) Send the request to the ONS, and return to IDS's URL;

3) The abattoir information server register registers to the IDS to indicate that it has the product information of traceability.

Process of product traceability:

4) Logistics and sales information server inquiries ONS by the product identifies, and returns with IDS's URL;

5) Query the IDS, and it returns with the URL of product information server of ownership, including the slaughterhouse information server URL.

6) Query the Information Server of slaughterhouse, and we can get the retrospective information of the product.

\section{Conclusion}

This paper takes GS1 standards and RFID identification technology and the characteristics of animal food industry chains into account, fully considers of the traceability systems at home and abroad, and takes their public properties, then proposes a general framework of the animal food traceability. In the framework, we used RFID tags, distributed information servers, Information Discovery Services and ONS, to complete the whole process of animal food tracing and tracking from farm to fork. And we have technically solved some urgent problems of animal food safety traceability systems, such as the Source Tracing, standardized, dynamic data sharing.

Data and management are key elements in food safety traceability, which calls for national legislation to change the management concept of enterprises. At present, some enterprises have taken action to products traceability management, but mostly limited to security, without using internationalGS1 standards in encoding. For tracking and tracing in the distribution center, supply chain, logistics and other links, it is necessary to promote international standards.

\section{Acknowledgements}

This work was supported by the National High-Tech Research and Development Plan of China under Grant No D07060501720702, 2006BAD22B06, 2006g35(3).We thank professor Zheng Limin, Zhu Hong, and Wu Ping for their helpful suggestions.

\section{References}

Cheung, A., Kailing, K., Schonauer, S.: Theseos: A Query Engine for Traceability across Sovereign, Distributed RFID Databases. IEEE Comm., 1495-1496 (2007)

Fabian, B., Günther, O., Spiekermann, S.: Security analysis of the Object Name Service for RFID. In: Workshop on Security, Privacy and Trust in Pervasive and Ubiquitous Computing (2005)

Xinxin, C., Subin, W., Shuo, W.: Radio Frequency Identification (RFID) technology and application system. Posts \& Telecom Press (2007)

Shanahan, C., Kernan, B., Ayalew, G., et al.: A framework for beef traceability from farm to slaughter using global standards: An Irish perspective. Computers and Electronics in Agriculture 66(1), 62-69 (2009) 
EPCglobal Inc. EPCglobal tag data standards v. 1.4. (June 2008), http: / /www. epcglobalinc.org/standards/tds / tds_1_4-standard-20080611.pdf

EPCglobal Inc. The EPCglobal architecture framework v.1.3. (March 2009), http: / /www.epcglobalinc.org/standards/architecture/ architecture_1_3-framework-20090319.pdf

GS1. GS1 Global Traceability Standard 1.1.0. (February 2009), http://www.gs1.org/docs/gsmp/traceability/ Global_Traceability_Standard.pdf

Brackenm, J., Mahtews, G.: GS1 Ireland Beef Traceability Case Study. GS1 Ireland (February 2005)

Hongliang, K., Jianhui, L.: Application Review of the Global Identification System (EAN.UCC System) in the Traceability of the Food Safety Supply Chain. Food Science 25(6), 188-194 (2004)

Jinyan, Z., Linli, T., Shizheng, G., et al.: Studies on Animal Food Safety Traceability System Using RFID Technology. Journal of Yunnan Agricultural University 23(4), 528-531 (2008)

Beier, S., Grandison, T., Kailing, K., et al.: Discovery Services-Enabling RFID Traceability in EPCglobal Networks. In: International Conference on Management of Data 2006, Delhi, India, December 14-16 (2006)

Linsen, Z., et al.: Design and Development of Quality Traceability Information Management System and Safety of the Beef Production's Entire Processes. Scientia Agricultura Sinica 39(10), 2083-2088 (2006) 